\title{
MR Imaging Diagnosis of Diencephalic-Mesencephalic Junction Dysplasia in Fetuses with Developmental Ventriculomegaly
}

\author{
(D) M. Severino, (D) A. Righini, (DD. Tortora, (D). Pinelli, (D) C. Parazzini, (D) G. Morana, DP. Accorsi, (D) V. Capra,
} (DD. Paladini, and (D) A. Rossi

\begin{abstract}
SUMMARY: Diencephalic-mesencephalic junction dysplasia is a rare malformation characterized by a poorly defined junction between the diencephalon and the mesencephalon, associated with a characteristic butterfly-like contour of the midbrain (butterfly sign). This condition may be variably associated with other brain malformations, including callosal abnormalities and supratentorial ventricular dilation, and is a potential cause of developmental hydrocephalus. Here, we have reported 13 fetuses with second-trimester obstructive ventriculomegaly and MR features of diencephalic-mesencephalic junction dysplasia, correlating the fetal imaging with available pathology and/or postnatal data. The butterfly sign can be clearly detected on axial images on fetal MR imaging, thus allowing for the prenatal diagnosis of diencephalic-mesencephalic junction dysplasia, with possible implications for the surgical management of hydrocephalus and parental counseling.
\end{abstract}

ABBREVIATION: DMJ = diencephalic-mesencephalic junction

$D^{i}$ iencephalic-mesencephalic junction (DMJ) dysplasia is a rare malformation characterized by a poorly defined junction between the diencephalon and the mesencephalon and associated with a characteristic butterfly-shaped contour of the midbrain on axial sections on MR imaging. ${ }^{1-3}$ This condition is included in a larger spectrum of DMJ anomalies, comprising forms that are recognizable in the axial (type A-DMJ anomalies, including DMJ dysplasia) or sagittal planes (type B-DMJ anomalies). ${ }^{3} \mathrm{DMJ}$ anomalies may be variably associated with other brain malformations, including callosal abnormalities and supratentorial ventricular dilation. In the original report, massively enlarged lateral ventricles were appreciated in 2 patients with DMJ dysplasia. ${ }^{1}$ Thereafter, partial aqueductal stenosis was identified in 1 patient with DMJ dysplasia and prenatal onset of severe ventriculomegaly, suggesting a mixed obstructive and malfor-

Received February 14, 2017; accepted after revision March 29.

From the Neuroradiology Unit (M.S., D.T., G.M., A. Rossi), Genetic Unit, Department of Neurosurgery (V.C.), and Fetal Medicine and Surgery Unit (D.P.), Istituto Giannina Gaslini, Genoa, Italy; Radiology and Neuroradiology Department (A. Righini, C.P.), Ospedale dei Bambini V Buzzi, Milano, Italy; and Neuroradiology (L.P.) and Neuropsychiatry Units (P.A.), Azienda Ospedaliera Spedali Civili di Brescia, Brescia, Italy.

Paper previously presented at: XX Symposium Neuroradiologicum, September 7-12, 2014; Istanbul, Turkey.

Please address correspondence to Mariasavina Severino, MD, Neuroradiology Unit, Istituto Giannina Gaslini, via Gaslini 5, Genoa 16147, Italy; e-mail: mariasavinaseverino@gaslini.org; @MSavinaSeverino; @TortoraDomenico

= Indicates article with supplemental on-line table.

Indicates article with supplemental on-line photo.

http://dx.doi.org/10.3174/ajnr.A5245 mative cause of the hydrocephalus. ${ }^{3}$ The prenatal imaging features of DMJ have not been reported. Moreover, the relationship between DMJ anomalies and early developmental hydrocephalus remains unclear.

Several causes of developmental hydrocephalus have been described in fetuses and children, mostly associated with myelomeningocele, aqueductal obstruction, posterior fossa crowding, and cysts or cephaloceles. ${ }^{4-6}$ To the best of our knowledge, no imaging features of DMJ dysplasia have been described in fetuses with developmental ventriculomegaly. Here, we have reported the second-trimester prenatal MR imaging of 13 fetuses with moderateto-severe obstructive ventriculomegaly presenting unequivocal features of DMJ dysplasia, and we correlated the imaging with available pathology and/or postnatal data.

\section{CASE SERIES}

This case series included 13 fetuses, referred to 3 pediatric institutions over a 10-year period (2005-2015) for fetal MR imaging because of second-trimester ultrasound findings of obstructive ventriculomegaly, in whom clear MR features of DMJ dysplasia were retrospectively detected. These cases were found by searching the fetal MR imaging data bases of the 3 centers for reports indicating obstructive ventriculomegaly, defined as bilateral atrial width $>12 \mathrm{~mm}$ with the hemisphere convexity reaching or almost reaching the skull inner surface, and with downward displacement of the fornix, without enlargement of the fourth ventricle. The DMJ dysplasia was not detected in the sonography examinations. 


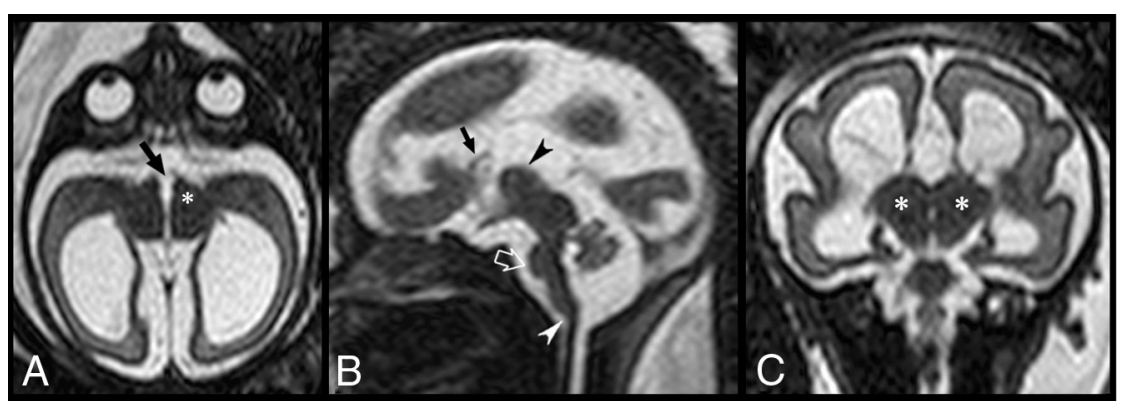

FIG 1. DMJ dysplasia in a 28-week-old fetus (case \#9). A, Axial T2-weighted image reveals fusion of the hypothalamus and midbrain (asterisk), enlargement of the dorsoventral axis of the midbrain, and a ventral midbrain cleft (arrow) resulting in a butterfly-like appearance. B, Sagittal T2weighted image demonstrates partial callosal agenesis (black arrow) associated with hypoplasia of the pons (empty arrow) and vermis and mild kinking of the cervicomedullary junction (white arrowhead). Note that the cerebral aqueduct is not visible. The interthalamic adhesion is enlarged and ventrally located (black arrowhead). C, Coronal T2-weighted image shows fusion between the midbrain and thalami (asterisks) as well as moderate supratentorial ventriculomegaly.

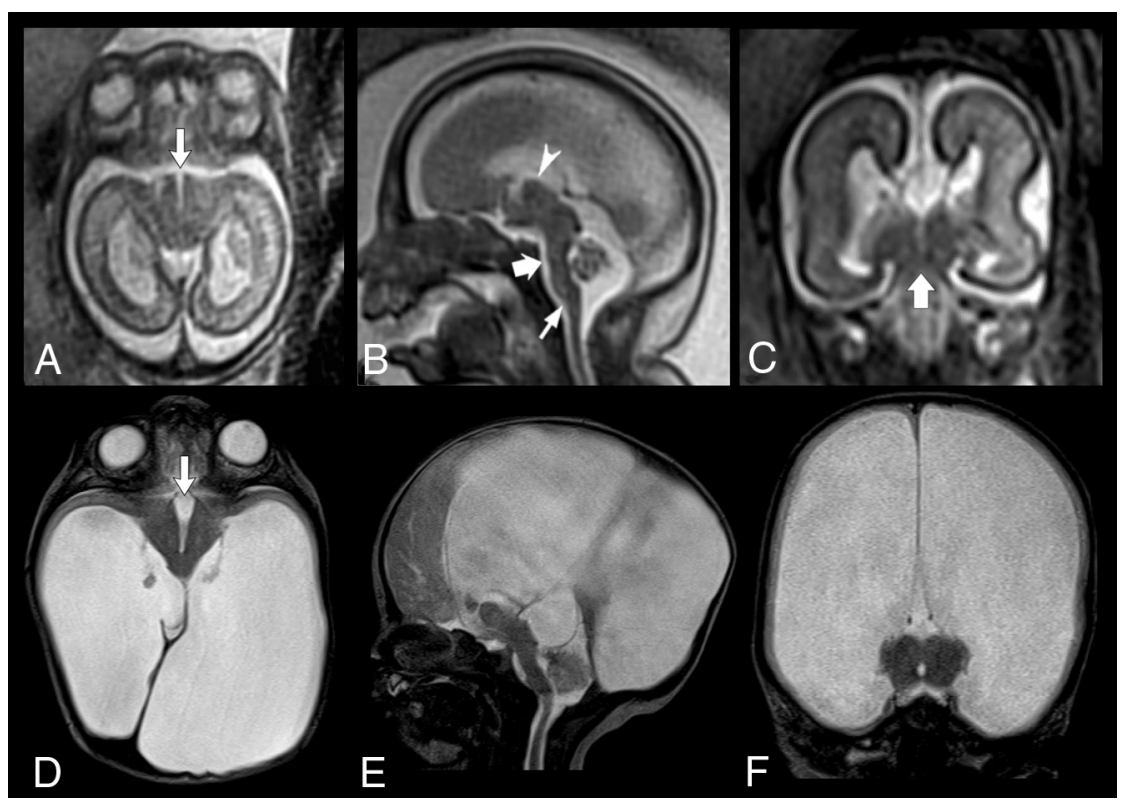

FIG 2. Prenatal and postnatal MR findings of DMJ dysplasia in a 23-week-old fetus (case \#7). A, Axial T2-weighted image reveals abnormal contour of the midbrain with a deep ventral cleft (arrow), resulting in a butterfly-like appearance. B, Sagittal T2-weighted image demonstrates marked hypoplasia of the pons (thick arrow) and vermis, aqueductal stenosis, and mild kinking of the cervicomedullary junction (thin arrow). The interthalamic adhesion is enlarged and ventrally located (arrowhead). C, Coronal T2-weighted image shows fusion between the midbrain and thalami (thick arrow) and complete callosal agenesis. Corresponding axial (D), sagittal (E) and coronal $(F)$ T2-weighted images obtained on day 5 after birth confirm the prenatal findings, including the DMJ dysplasia (arrow, D) and brain stem anomalies, and reveal severe supratentorial hydrocephalus.

The MR imaging studies were performed for clinical purposes on 1.5T scanners (Achieva, Philips Healthcare, Best, the Netherlands; Ingenia, Philips Healthcare; Magnetom Avanto, Siemens, Erlangen, Germany) with a phased array abdominal or cardiac coil after an expert neurosonography study. All studies included 3 mm-thick single-shot fast spin-echo multiplanar T2-weighted images. The mothers had signed a specific consent form for fetal MR imaging, and the study complied with the internal guidelines for retrospective studies of the 3 institutions.

The On-line Table summarizes the clinical radiologic data of all fetuses. The mean gestational age at first fetal MR imaging was 23.8 weeks (range, 20-31 weeks). All fetuses were males. The mean maternal age was 32.9 years. Congenital infections and alcohol abuse were excluded in all pregnancies. No karyotype anomalies were reported in the $11 / 13$ cases with available testing.

The mean atrial width was $20.6 \mathrm{~mm}$ (range, 12-38 $\mathrm{mm}$ ). Supratentorial brain mantle thinning was noted in all fetuses, with the brain surface reaching or almost reaching the inner skull surface. Complete or partial agenesis of the corpus callosum was noted in 9/13 fetuses, and marked callosal thinning caused by the underlying ventriculomegaly was seen in the remaining 4 cases. In all cases, on prenatal MR imaging, the butterfly sign was detected on axial sections, and on sagittal images, a poorly defined DMJ was noticeable, with the thalamic mass sitting in an abnormal anterior position with respect to the midbrain (Fig 1 and On-line Figure). On coronal sections, a merger between the midbrain and thalami was detected in all cases. Ventral dislocation of an enlarged interthalamic adhesion was noted in 9/13 fetuses, and the aqueduct was poorly defined in 10/13 cases. Pontine hypoplasia was identified in 6/13 fetuses and cerebellar hypoplasia in 3 cases. A moderate brain stem kinking was present in $11 / 13$ cases.

In $9 / 13$ cases, the parents elected for termination of pregnancy after prenatal counseling. Two fetuses had a second antenatal MR imaging performed 1 and 10 weeks after the first examination, respectively. Postnatal MR imaging was available in 4 patients (Fig 2), and MR autopsy was performed in 1 fetus. DTI was performed in 2 patients on a $1.5 \mathrm{~T}$ scanner along 34 noncolinear directions by using an axial singleshot spin-echo echo-planar sequence, with b-values of 0 and $1000 \mathrm{~s} / \mathrm{mm}^{2}$; TR, $9203 \mathrm{~ms}$; TE, $71 \mathrm{~ms}$; section thickness, $2 \mathrm{~mm}$; FOV, $240 \times 240 \mathrm{~mm}$; matrix size, $128 \times 128$. The corticospinal tracts were reconstructed with a deterministic algorithm by using the IntelliSpace Portal 8.0 (Philips Healthcare). Interestingly, no tracking of corticospinal tracts was obtained below the level of the midbrain (Fig 3). Neuropathologic data were available in 2 fetuses, confirming the altered DMJ segmentation with deep ventral midbrain cleft and the level and etiology of the obstruction. In 1 fetus, there was a trifurcation of the cerebral aqueduct, and the olivary nuclei were small and anteriorly dislocated. Moreover, an incomplete lobulation of the right lung and right-sided moderate hydroureteronephrosis were noted. Overall, the prenatal diagno- 


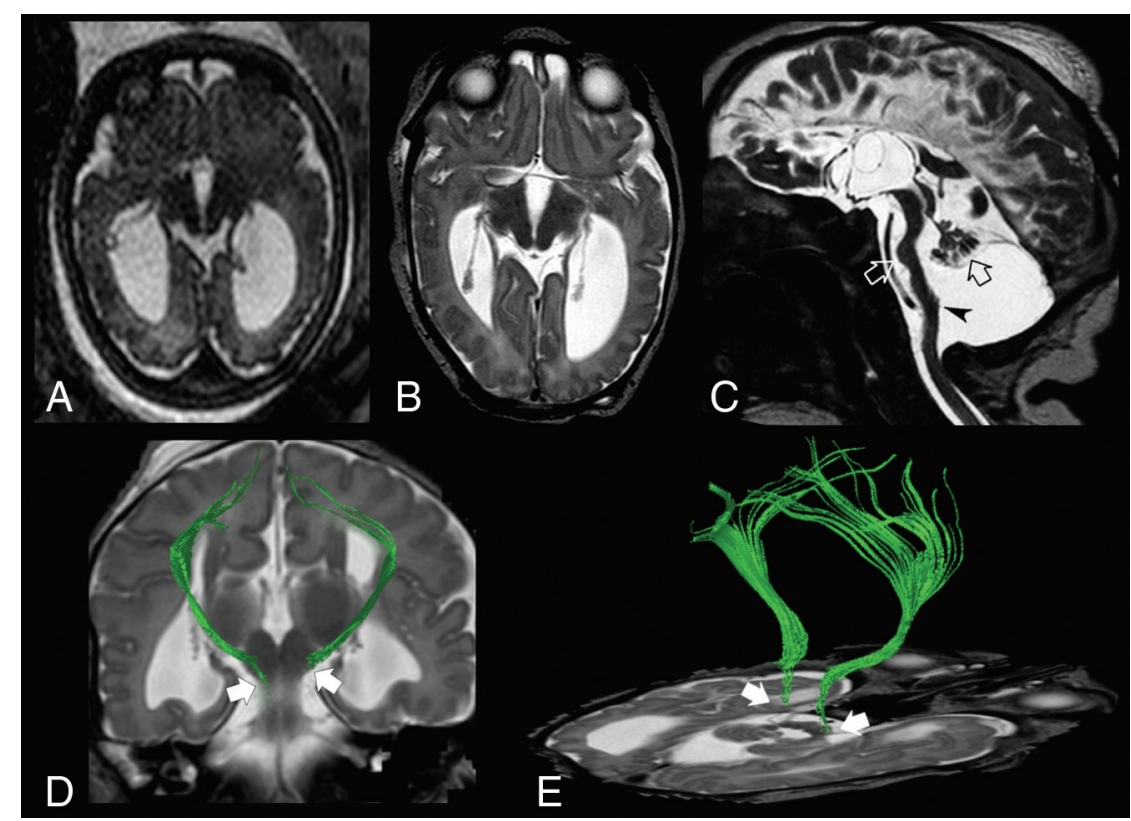

FIG 3. Prenatal and postnatal MR findings with DTI in a 31-week-old fetus with DMJ dysplasia (case \#13). A, Axial T2-weighted image shows the typical "butterfly" appearance of the DMJ. B, Axial T2-weighted image obtained at age 7 days confirms the abnormal contour of the midbrain. C, Sagittal T2-weighted driven equilibrium pulse image demonstrates complete callosal agenesis, marked hypoplasia of the pons (white empty arrow) and vermis (black empty arrow), elongated midbrain, and kinked cervicomedullary junction (arrowhead). Coronal (D) and oblique axial (E) T2-weighted images fused with motor tractography reveal no tracking of the corticospinal tracts below the level of the midbrain (thick arrows). Color version available on-line.

sis of DMJ dysplasia was confirmed postnatally or ex vivo in $6 / 13$ cases of this series.

\section{DISCUSSION}

We described a relatively large series of fetuses with second-trimester obstructive ventriculomegaly associated with clear features of DMJ dysplasia. Our cohort demonstrates that DMJ dysplasia could already be detected before gestational week 24 and that it has to be included among the causes of early developmental hydrocephalus. Fetal ventriculomegaly is a relatively common finding, occurring in approximately $1 \%$ of fetuses on sonography and in 1 of 1000 live born neonates. ${ }^{4}$ Conversely, DMJ dysplasia is probably rare, though it may be underestimated in cases without ventriculomegaly. The role of MR imaging in detecting brain anomalies in fetuses with ventriculomegaly is well established. Griffiths et $\mathrm{al}^{7}$ demonstrated a $17 \%$ risk of finding other brain abnormalities in fetuses with a confident sonography diagnosis of isolated ventriculomegaly. More recently, Barzilay et $\mathrm{al}^{8}$ showed that in fetuses with ventriculomegaly, the MR detection rate of minor and major brain findings increases with each millimeter increase in ventricle width. Various etiologies of fetal ventriculomegaly have been demonstrated, including acquired causes such as intraventricular hemorrhage, infection, or neoplasms. ${ }^{4,5}$ When these have been excluded, radiologists must carefully assess MR images to identify additional brain abnormalities that may suggest a developmental/malformative cause of the hydrocephalus. ${ }^{9}$

DMJ dysplasia is a rare malformation first described in 6 children born from 3 consanguineous Egyptian families, with facial dysmorphisms, severe cognitive impairment, axial hypotonia, spastic quadriparesis, vasomotor instability, unexplained fever, and seizures. ${ }^{1}$ So far, no causative genes have been linked to this condition. On imaging, DMJ dysplasia is characterized by a poorly defined junction between the diencephalon and the mesencephalon and by a ventral cleft contiguous with the third ventricle, producing a characteristic butterfly-shaped contour of the midbrain on axial images. ${ }^{1-3}$ Notably, MR imaging identified the butterfly sign and the abnormal relation between the midbrain and thalamic mass already at 20-21 gestational weeks in 6 fetuses of this series. Nonetheless, if not carefully sought, this finding may be overlooked, especially in the early gestational weeks. Therefore, to improve the detection rate of DMJ anomalies in earlier gestational stages, fetal MR imaging must be technically adequate, with perfectly oriented axial planes, and should not be hampered by fetal motion artifacts.

Developmental forms of hydrocephalus often have multiple points of obstruction. ${ }^{4}$ In the present series, cerebral aqueduct stenosis was confirmed in all cases with postnatal MR imaging or autopsy. Moreover, the cerebral aqueduct was scarcely visible in most fetuses of our series, suggesting that aqueductal stenosis could be the main cause of obstructive ventriculomegaly. Of note, the identification of the lumen of the cerebral aqueduct on MR imaging may not always be reliable in the early gestational weeks, often requiring follow-up studies for confirmation. Interestingly, in most of the present fetuses, the interthalamic adhesion was additionally enlarged and caudally displaced. The location of the interthalamic adhesion is highly variable in humans, but in most people, it lies at the center or in the anterior upper quadrant of the third ventricle, whereas it is located in the posterior inferior quadrant in fewer than $1 \%$ of cases. ${ }^{10}$ A recent computational study on CSF dynamics demonstrated that pressure in the third ventricle is higher when the interthalamic adhesion is located close to the cerebral aqueduct. ${ }^{11}$ Partial or complete aqueductal stenosis associated with alterations of CSF flow dynamics within the third ventricle and abnormal WM development might thus explain the ventriculomegaly in these fetuses. In addition, the interthalamic adhesion hypertrophy is an important dysmorphic feature reported in several brain malformations, including Chiari II malformation, L1 syndrome with X-linked hydrocephalus, ${ }^{12}$ and $6 \mathrm{q}$ terminal deletion syndrome. ${ }^{13}$ Notably, marked interthalamic adhesion hypertrophy may overlap with diencephalosynapsis, a rare malformation designating a complete or partial fusion of the thalami associated with secondary reduction of the lumen of the third ventricle, ${ }^{14,15}$ and with rhombencephalosynapsis. ${ }^{6}$

Interestingly, all our presented fetuses were males, suggesting a possible X-linked inheritance pattern. Neuropathologic data from 138 fetuses and neonates genetically tested for X-linked 
hydrocephalus showed that 56 subjects (42\%) harbored L1CAM gene mutations, and the remaining fetuses (58\%) were classified into 4 distinct subgroups: 1) "L1-like" syndrome, including fetuses with no L1CAM mutations, but exhibiting characteristics of L1 syndrome (20\%); 2) aqueductal atresia/forking spectrum, often associated with midbrain-hindbrain dorsoventral patterning defects $(27 \%)$; 3 ) hydrocephalus associated with polymalformative syndromes, such as VACTERL-H (9\%); and 4) hydrocephalus associated with isolated CNS malformations (44\%). ${ }^{16}$ The L1CAM gene (Xq28) encodes a highly conserved type 1 transmembrane protein of the immunoglobulin superfamily that plays important roles in neuronal adhesion, neuronal migration, axonal growth, pathfinding, and fasciculation as well as in the development of the ventricular system and cerebellum. On imaging, L1 syndrome with X-linked hydrocephalus is usually characterized by bi- or triventricular dilation, aqueductal stenosis, enlarged quadrigeminal plate, interthalamic adhesion hypertrophy, and vermian hypoplasia. ${ }^{12,17}$ Intriguingly, most of the fetuses in our series showed phenotypic and imaging characteristics similar to L1CAM-mutated fetuses. Moreover, postnatal MR imaging with DTI revealed corticospinal tract abnormalities in 2 cases, likely corresponding to the agenesis/fragmentation of the corticospinal tracts described in neuropathology cases of L1 and L1-like syndromes. Unfortunately, because of the lack of L1CAM genetic analysis in the present cases, we cannot establish whether DMJ dysplasia is caused by L1CAM mutations or if it belongs to the L1-like spectrum. Moreover, data on fetal MR features of L1 or L1-like syndrome are not available in the literature. DMJ features are likely to occur on a different genetic basis, and studies on genotype-phenotype correlation are definitively needed.

Finally, we observed pontine hypoplasia and small cerebellar vermis in most of the present fetuses, variably associated with moderate brain stem kinking. The differential diagnosis of prenatal ventriculomegaly and brain stem kinking has been recently widened to include several brain malformations, such as congenital muscular dystrophies, X-linked hydrocephalus caused by L1CAM mutations, microcephaly with lissencephaly and mid-hindbrain involvement, and tubulinopathies. ${ }^{18,19}$ Taken together, these data indicate that a kinked brain stem is not a pathognomonic finding, but more likely an indicator of severe neurodysgenesis arising early in gestation and often associated with developmental hydrocephalus. ${ }^{18}$

The main limitation of our report is the lack of extensive genetic testing and histology investigation in all cases with pregnancy termination. Moreover, the possibility that fetal DMJ dysplasia cases without developmental obstructive ventriculomegaly may have been missed in our prenatal imaging search has to be taken into account.

In conclusion, a butterfly-shaped contour of the midbrain along with an abnormal spatial relation between the midbrain and thalamic mass may be detected on axial and sagittal sections, respectively, thus allowing an early prenatal diagnosis of DMJ dysplasia in fetuses with proximal obstructive ventriculomegaly. Among the rare causes of developmental hydrocephalus, DMJ dysplasia is likely to be an underestimated condition and seems to share several phenotypic features with the L1 and L1-like syndromes. Further studies on larger fetal populations with genotype-phenotype correlations are needed to clarify the causes, pathophysiology, and prevalence of DMJ dysplasia in the fetal population.

Disclosures: Andrea Rossi—UNRELATED: Consultancy: Bracco Imaging Italia SrL.

\section{REFERENCES}

1. Zaki MS, Saleem SN, Dobyns WB, et al. Diencephalic-mesencephalic junction dysplasia: a novel recessive brain malformation. Brain 2012;135:2416-27 CrossRef Medline

2. Jissendi-Tchofo P, Severino M, Nguema-Edzang B, et al. Update on neuroimaging phenotypes of mid-hindbrain malformations. $\mathrm{Neu}$ roradiology 2015;57:113-38 CrossRef Medline

3. Severino M, Tortora D, Pistorio A, et al. Expanding the spectrum of congenital anomalies of the diencephalic-mesencephalic junction. Neuroradiology 2016;58:33-44 CrossRef Medline

4. Tully HM, Dobyns WB. Infantile hydrocephalus: a review of epidemiology, classification and causes. Eur J Med Genet 2014;57:359-68 CrossRef Medline

5. Tully HM, Ishak GE, Rue TC, et al. Two hundred thirty-six children with developmental hydrocephalus: causes and clinical consequences. J Child Neurol 2016;31:309-20 CrossRef Medline

6. Cagneaux M, Vasiljevic A, Massoud M, et al. Severe second-trimester obstructive ventriculomegaly related to disorders of diencephalic, mesencephalic and rhombencephalic differentiation. Ultrasound Obstet Gynecol 2013;42:596-602 CrossRef Medline

7. Griffiths PD, Reeves MJ, Morris JE, et al. A prospective study of fetuses with isolated ventriculomegaly investigated by antenatal sonography and in utero MR imaging. AJNR Am J Neuroradiol 2010; 31:106-11 CrossRef Medline

8. Barzilay E, Bar-Yosef O, Dorembus S, et al. Fetal brain anomalies associated with ventriculomegaly or asymmetry: an MRI-based study. AJNR Am J Neuroradiol 2017;38:371-75 CrossRef Medline

9. Huisman TAGM, Tekes A, Poretti A. Brain malformations and fetal ventriculomegaly: what to look for? J Pediatr Neuroradiol 2012;1: 185-95 CrossRef

10. Samra KA, Cooper IS. Radiology of the massa intermedia. Radiology 1968;91:1124-28 CrossRef Medline

11. Cheng S, Tan K, Bilston LE. The effects of the interthalamic adhesion position on cerebrospinal fluid dynamics in the cerebral ventricles. J Biomech 2010;43:579-82 CrossRef Medline

12. Kanemura $Y$, Okamoto N, Sakamoto $H$, et al. Molecular mechanisms and neuroimaging criteria for severe L1 syndrome with $\mathrm{X}$-linked hydrocephalus. J Neurosurg 2006;105(5 suppl):403-12 CrossRef Medline

13. Elia M, Striano P, Fichera M, et al. 6q terminal deletion syndrome associated with a distinctive EEG and clinical pattern: a report of five cases. Epilepsia 2006;47:830-38 CrossRef Medline

14. De France I, Saada P, Jouannic JM, et al. Ultrasonographic and pathological correlation in a fetal intracranial cyst: a case of "diencephalo-synapsis" [in French]. J Gynecol Obstet Biol Reprod (Paris) 2002;31:600-03 Medline

15. Kepes JJ, Clough C, Villanueva A. Congenital fusion of the thalami (atresia of the third ventricle) and associated anomalies in a 6-monthsold infant. Acta Neuropathol 1969;13:97-104 CrossRef Medline

16. Adle-Biassette H, Saugier-Veber P, Fallet-Bianco C, et al. Neuropathological review of 138 cases genetically tested for $\mathrm{X}$-linked hydrocephalus: evidence for closely related clinical entities of unknown molecular bases. Acta Neuropathol 2013;126:427-42 CrossRef Medline

17. Yamasaki M, Kanemura Y. Molecular biology of pediatric hydrocephalus and hydrocephalus-related diseases. Neurol Med Chir (Tokyo) 2015;55:640-46 CrossRef Medline

18. Stroustrup Smith A, Levine D, Barnes PD, et al. Magnetic resonance imaging of the kinked fetal brain stem: a sign of severe dysgenesis. $J$ Ultrasound Med 2005;24:1697-709 CrossRef Medline

19. Amir T, Poretti A, Boltshauser E, et al. Differential diagnosis of ventriculomegaly and brainstem kinking on fetal MRI. Brain Dev 2016; 38:103-08 CrossRef Medline 